Proc. 15th Int. Conference on Defects Recognition, Imaging and Physics in Semiconductors, Warsaw, Poland 2013

\title{
Photoluminescence Analysis of Oxygen Precipitation around Small-Angle Grain Boundaries in Multicrystalline Silicon Wafers
}

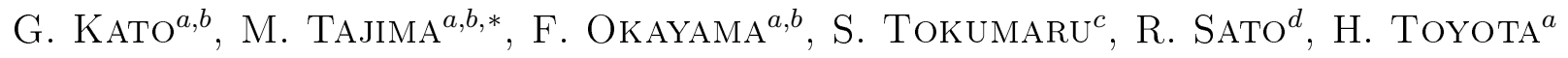 \\ AND A. OGURA ${ }^{b}$ \\ ${ }^{a}$ Institute of Space and Astronautical Science/JAXA, Sagamihara 252-5210, Japan \\ ${ }^{b}$ Meiji University, Kawasaki 214-8571, Japan \\ ${ }^{c}$ Nippon Steel \& Sumitomo Metal Corporation, Futtsu 293-8511, Japan \\ ${ }^{d}$ Nippon Steel \& Sumikin Technology Co., Ltd, Futtsu 293-8511, Japan
}

\begin{abstract}
We have investigated the correlation between deep-level photoluminescence and the density of small-angle grain boundaries in multicrystalline $\mathrm{Si}$. A deep-level photoluminescence component around $0.87 \mathrm{eV}$, which we previously ascribed to oxygen precipitates, became lower and higher in the region with high and low density of small-angle grain boundaries, respectively. This can be explained by the differences in the availability of oxygen atoms around respective small-angle grain boundaries. We performed focused ion beam time-of-flight secondary ion mass spectroscopy on special points emitting extremely strong $0.87 \mathrm{eV}$ emission, and detected a clustered area of ${ }^{16} \mathrm{O}^{-}$. This is strong evidence for the idea that the $0.87 \mathrm{eV}$ band is due to oxygen precipitates.
\end{abstract}

DOI: 10.12693/APhysPolA.125.1010

PACS: 78.55.Ap, 61.72.Qq, 82.80.Rt, 88.40.jj

\section{Introduction}

Multicrystalline $\mathrm{Si}$ (mc-Si) is advantageous for the mass production of low-cost solar cells. However, mc-Si contains numerous small-angle grain boundaries (SAGBs), which act as recombination centers and decrease the minority carrier lifetime [1-4]. This is the reason that the conversion efficiency of mc-Si cells is lower than that of single crystalline Si cells. Thus, extensive characterization of SA-GBs in mc-Si has been required. Photoluminescence (PL) intensity mapping is a useful technique to examine the distribution of SA-GBs non-destructively and without contact. SA-GBs appeared as dark lines in PL intensity mapping of the band-to-band (BB) emission at $1.1 \mathrm{eV}[5,6]$. In contrast, the intensity of the deep-level emissions became strong around SA-GBs. We reported that deep-level emissions at room temperature consist of multiple bands [7]. One deep-level PL labeled $D_{\mathrm{b}}$ around $0.87 \mathrm{eV}$ was ascribable to oxygen precipitates. The intensity of the $D_{\mathrm{b}}$ band became higher on SA-GBs, reflecting that these grain boundaries acted as preferential precipitation sites for oxygen [7]. Another deep-level PL labeled $D_{\mathrm{a} 1}$ around $0.79 \mathrm{eV}$, which was attributable to secondary defects or impurities attracted by a strain field around SA-GBs, appeared as bright bands surrounding SA-GBs [7]. Objectives of the present study were to investigate the relationship between these deep-level emissions and the density of SA-GBs and to identify the origin of the $D_{\mathrm{b}}$ band emission.

*corresponding author; e-mail: tajima@isas.jaxa.jp

\section{Experimental technique}

Samples used for the present study were mc-Si wafers with a size of $156 \times 156 \mathrm{~mm}^{2}$ and thickness of $170 \mu \mathrm{m}$ from a slowly solidified and a rapidly solidified ingot, where the ratio of the solidified rate between the two ingots was about 1.5. The slicing damage was etched off by a $\mathrm{HNO}_{3} / \mathrm{HF}$ solution. PL mapping experiments were performed at room temperature [8] under the excitation of the $532 \mathrm{~nm}$ line of a diode-pumped solid-state laser. The excitation power was $2-30 \mathrm{~mW}$ with a beam diameter of about $10 \mu \mathrm{m}$ on the sample surface. The intervals of data sampling were 5 and $20 \mu \mathrm{m}$ in Figs. 1 and 3, respectively. Emission from the samples was passed through bandpass filters to extract specific spectral components of interest, and then detected by a cooled photomultiplier (Hamamatsu R5509-72). To identify species of impurities in a minute area, we analyzed samples by a focused ion beam time-of-flight secondary ion mass spectrometer (FIB-TOF-SIMS).

\section{Results and discussion}

The microscopic intensity patterns for the $D_{\mathrm{a} 1}$ emission at $0.79 \mathrm{eV}, D_{\mathrm{b}}$ emission at $0.87 \mathrm{eV}$ and $\mathrm{BB}$ emission at $1.1 \mathrm{eV}$ in wafers from the slowly solidified ingot are shown in Fig. 1. The density of SA-GBs in the area of Fig. 1a-c is higher than in Fig. 1d-f, where the density was estimated from dark lines in the BB emission in Fig. $1 \mathrm{c}$ and $\mathrm{f}$. The difference in the density of SA-GBs caused the change of the intensity of the $D_{\mathrm{b}}$ band emission, as shown in Fig. $1 \mathrm{~b}$ and e. The intensity became lower and higher in the region with high and low density of SA-GBs, respectively. This can be explained by 


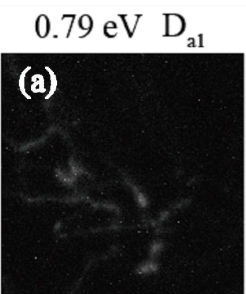

$0.87 \mathrm{eV} \quad \mathrm{D}$
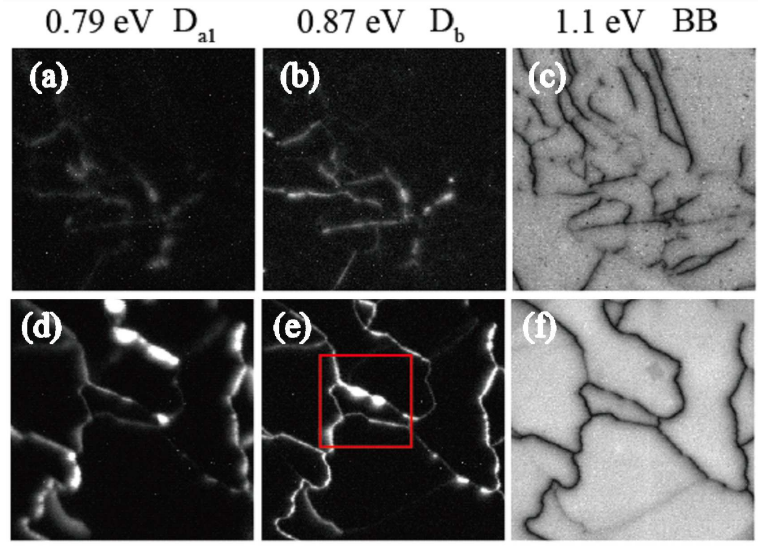

High

density

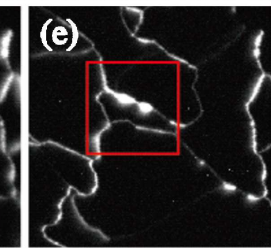

Low

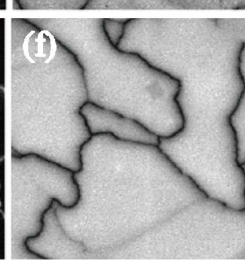

High

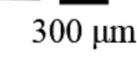

Fig. 1. Microscopic intensity patterns for $D_{\mathrm{a} 1}$ emission at $0.79 \mathrm{eV}, D_{\mathrm{b}}$ emission at $0.87 \mathrm{eV}$ and $\mathrm{BB}$ emission at $1.1 \mathrm{eV}$ in slowly solidified wafers. Density of SA-GBs in the area of $(\mathrm{a})-(\mathrm{c})$ is higher than in $(\mathrm{d})-(\mathrm{f})$.

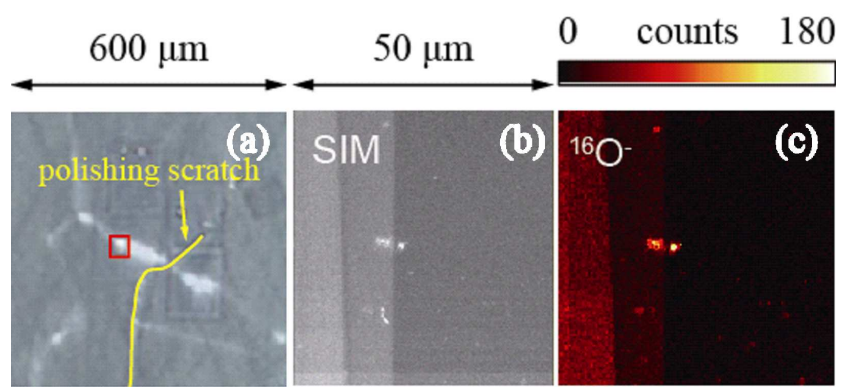

Fig. 2. (a) Intensity mapping of $D_{\mathrm{b}}$ band on the region marked with a square in Fig. 1e, overlapping on optical micrograph. (b) SIM image of the area marked with a square in (a). (c) FIB-TOF-SIMS image of ${ }^{16} \mathrm{O}^{-}$in the same region as (b).
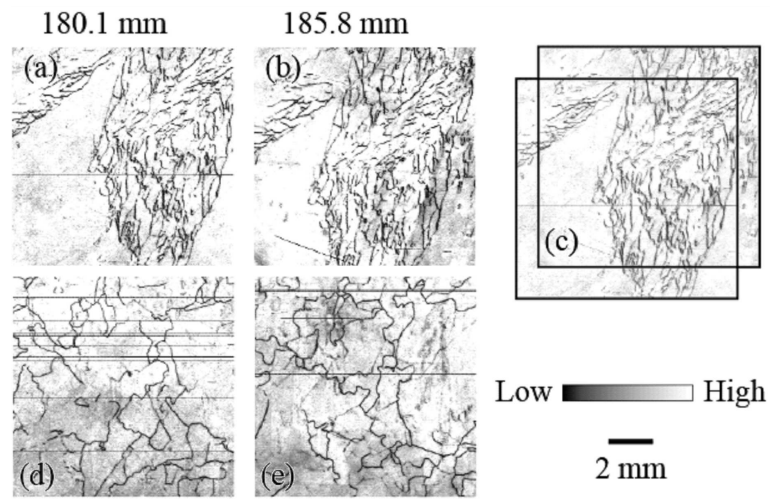

High density

Fig. 3. Intensity mappings of BB band representing SA-GB patterns of (a) high and (d) low density area at a height of $180.1 \mathrm{~mm}$. Identical high and low areas at a height of $185.8 \mathrm{~mm}$ are shown in (b) and (e). Patterns in (a) and (b) are overlapped in (c). the hypothesis that SA-GBs act as preferential precipitation sites for oxygen and a shortage of oxygen occurs in an area where their density is high, causing oxygen precipitation retardation.

The intensity pattern for the $D_{\mathrm{a} 1}$ band was also studied as shown in Fig. 1a, d. The intensity became higher in the region with lower density of SA-GBs. This can be explained in the same way as in the $D_{\mathrm{b}}$ band: the concentration of the secondary defects or impurities interacting with SA-GBs becomes higher in an area where SA-GBs density is lower.

In order to check the validity of the above-mentioned hypothesis, we calculated the diffusion length of oxygen during the crystal growth. The diffusion constant of oxygen, $D_{\mathrm{O}}$, which is not affected by oxygen density, crystallographic orientation, or dopant impurities in a wide temperature range is given by

$$
D_{\mathrm{O}}=0.13 \exp (-2.53 \mathrm{eV} / \mathrm{kT}),
$$

where $k$ is the Boltzmann constant [9]. The diffusion length is given by

$$
L=2 \sqrt{D t},
$$

where $t$ is the diffusion time. As a typical example, mcingots stay in an ambient higher than $1500 \mathrm{~K}$ for $2 \mathrm{~h}[10]$. In this case the diffusion length of oxygen is calculated to be more than $160 \mu \mathrm{m}$. Since the value is comparable to the spacing of neighboring SA-GBs in Fig. 1c, we believe that the oxygen diffusion is one important factor of the present oxygen precipitation process.

The results of PL mappings for wafers sliced from the rapidly solidified ingot were essentially the same as those for the slowly solidified samples. The crucial difference was the presence of points exhibiting extremely strong $D_{\mathrm{b}}$ emission in slowly solidified samples as shown in a squared area in Fig. 1e. We believe that this reflects the large growth of oxygen precipitates during a long period at high temperature in the slow solidification process.

We performed FIB-TOF-SIMS analysis on the strong intensity points in the square in Fig. 1e. The first step was to determine the target points for the analysis. An optical micrograph around the points was overlapped on the highly spatially resolved intensity mapping of the $D_{\mathrm{b}}$ band, as shown in Fig. 2a. A polishing scratch was used as a guide for determining the point. We found minute foreign substances by scanning ion microscope (SIM) image, as shown in Fig. 2b. Then, we removed oxidation films by sputtering and performed FIB-TOF-SIMS analysis of the point. As shown in Fig. 2c, the signal of ${ }^{16} \mathrm{O}^{-}$ was apparently higher on the foreign substances. The same intensity pattern was observed for $\mathrm{SiO}_{2}^{-}$, while no substantial variations around the substances were recognized for ${ }^{27} \mathrm{Al}^{+},{ }^{12} \mathrm{C}^{-}$, or ${ }^{35} \mathrm{Cl}^{-}$. This is strong evidence for the idea that the foreign substances responsible for the $D_{\mathrm{b}}$ band are oxygen precipitates.

Finally, we consider it worthwhile to point out the difference in the propagation of SA-GBs between the areas where they are high and low in density. Figure 3 shows the intensity mappings of the $\mathrm{BB}$ band representing the 
SA-GB patterns in (a) high and (d) low density areas in the wafer sliced from the rapidly solidified ingot at a height of $180.1 \mathrm{~mm}$ from the bottom, where the total height of the ingot is about $240 \mathrm{~mm}$. The identical high and low density areas at a height of $185.8 \mathrm{~mm}$ are shown in (b) and (e), respectively. The SA-GB patterns propagated parallel to the growth direction in the high density area as shown in Fig. 3c, where the patterns in (a) and (b) are overlapped. This indicates that the SA-GBs have a columnar structure. In contrast, the patterns in the low density area varied complicatedly with the growth direction, as shown in Fig. 3d and e. Our group reported previously a similar variation of the SA-GB structure and discussed its origin in relation to the crystal growth condition [5].

\section{Conclusions}

We confirm the origin of the $D_{\mathrm{b}}$ band appearing on SAGBs in mc-Si as oxygen precipitates on the basis of the microscopic deep-level PL mapping and FIB-TOF-SIMS analysis. The intensity of the $D_{\mathrm{b}}$ band became higher in the region with a lower density of SA-GBs, suggesting that more oxygen atoms were available for the precipitation at respective grain boundaries. The $D_{\mathrm{b}}$ band appeared granular along SA-GBs and especially strong intensity points were often observed in slowly solidified samples but not in rapidly solidified samples. This reflects that the long-time annealing enhances the oxygen precipitation in the slowly solidifying process. Finally, oxygen ions were successfully detected from the strong intensity points by FIB-TOF-SIMS analysis. These results allow us to conclude definitely that the $D_{\mathrm{b}}$ band is due to oxygen precipitates.

\section{Acknowledgments}

This work was supported by the New Energy and Industrial Technology Development Organization (NEDO) under the Ministry of Economy, Trade and Industry of Japan.

\section{References}

[1] J. Chen, T. Sekiguchi, R. Xie, P. Ahmet, T. Chikyo, D. Yang, S. Ito, F. Yin, Scr. Mater. 52, 1211 (2005).

[2] H. Sugimoto, M. Inoue, M. Tajima, A. Ogura, Y. Oshita, Jpn. J. Appl. Phys. 45, L641 (2006).

[3] W. Seifert, G. Morgenstern, M. Kittler, Semicond. Sci. Technol. 8, 1687 (1993).

[4] Y. Ohshita, Y. Nishikawa, M. Tachibana, V.K. Tuong, T. Sasaki, N. Kojima, S. Tanaka, M. Yamaguchi, J. Cryst. Growth 275, e491 (2005).

[5] H. Sugimoto, K. Araki, M. Tajima, T. Eguchi, I. Yamaga, M. Dhamrin, K. Kamisako, T. Saitoh, J. Appl. Phys. 102, 054506 (2007).

[6] T. Trupke, R.A. Bardos, M.D. Abbott, P. Würfel, E. Pink, Y. Augarten, F.W. Chen, K. Fisher, J.E. Cotter, M. Kasemann, M. Rüdiger, S. Kontermann, M.C. Schubert, M. The, S.W. Glunz, W. Warta, D. Macdonald, J. Tan, A. Cuevas, J. Bauer, R. Gupta, O. Breitenstein, T. Buonassisi, G. Tarnowski, A. Lorenz, H.P. Hartmann, D.H. Neuhaus, J.M. Fernandez, in: Proc. 22nd Europ. Photovoltaic Solar Energy Conf., Milan (Italy), 2007, WIP-Renewable Energies, München 2007, p. 22.

[7] M. Tajima, Y. Iwata, F. Okayama, H. Toyota, H. Onodera, T. Sekiguchi, J. Appl. Phys. 111, 113523 (2012).

[8] M. Tajima, Z. Li, R. Shimidzu, Jpn. J. Appl. Phys. 41, L1505 (2002).

[9] J.C. Mikkelsen, Jr., in: Oxygen, Carbon, Hydrogen and Nitrogen in Crystalline Silicon, Eds. J.C. Mikkelsen, Jr., S. Paearton, J.W. Corbett, S.J. Pennycook, Mater. Res. Soc., Pittsburgh 1986, p. 19.

[10] S. Nakano, X.J. Chen, B. Gao, K. Kakimito, J. Cryst. Growth 318, 280 (2011). 